[0212-7199 (2007) 24: 2; pp 57-60] ANALES DE MEDICINA INTERNA Copyright (C) 2007 ARAN EDICIONES, S.L.

AN. MED. INTERNA (Madrid) Vol. 24, N. ${ }^{\circ} 2$, pp. 57-60, 2007

\title{
Estudio de la calidad de vida de pacientes con insuficiencia cardiaca en un Servicio de Medicina Interna
}

\author{
D. ETXEBERRÍA-LEKUONA, J. SÁNCHEZ-ÁLVAREZ, A. ALONSO GUTIÉRREZ', \\ V. ACHA ARRIETA, R. CAMPOS RIVAS, V. JARNE BETRÁN \\ Servicio de Medicina Interna y ${ }^{\prime}$ Medicina Preventiva. Hospital Virgen del Camino. \\ Pamplona
}

STUDY OF QUALITY OF LIFE OF PATIENTS WITH HEART FAILURE IN AN INTERNAL MEDICINE DEPARTMENT

\begin{abstract}
RESUMEN
Introducción: la calidad de vida (CV) es un objetivo importante del tratamiento de los pacientes con insuficiencia cardiaca (IC). Hemos medido la $\mathrm{CV}$ en una muestra de pacientes ingresados que presentan IC y diversos factores que pueden influir en ella.

Métodos: se evaluó a 34 pacientes con IC utilizando un cuestionario específico, el Minnesota Living With Heart Failure Questionnaire (MLWHFQ). Además se realizaron mediciones analíticas y de ecocardiografía y se aplicaron cuestionarios específicos con el fin de evaluar otros parámetros que pueden influir en la CV: la edad, el sexo, la comorbilidad, la medicación habitual, la fracción de eyección del ventrículo izquierdo, el estado nutricional, la función cognitiva, la depresión y la capacidad funcional en la vida diaria.

Resultados: la CV medida mediante el cuestionario MLWHFQ de los pacientes del estudio fue alta (media: 34,3 ). Los otros tests realizados dieron valores dentro de la normalidad o próximos a ella. La depresión fue el factor que con más claridad se asoció con la CV de los pacientes $(\mathrm{p}=0,0001)$. También demostraron una asociación estadísticamente significativa la fracción de eyección del ventrículo izquierdo $(\mathrm{p}=0.03)$, la edad $(\mathrm{p}=0,008)$ y la albuminemia $(\mathrm{p}=0,01)$.

Conclusiones: En nuestra muestra, la CV de los pacientes estaba condicionada por la depresión, la fracción de eyección del ventrículo izquierdo, la edad y la albuminemia.
\end{abstract}

PALABRAS CLAVE: Insuficiencia cardiaca. Calidad de vida. Minnesota.

\section{ABSTRACT}

Introduction: Quality of life $(Q L)$ is an important objective in the treatment of patients with heart failure $(H F)$. We have measured $Q L$ and different factors that can affect it in a group of patients hospitalized with $H F$.

Methods: 34 patients with $H F$ were cross-examined by means of a specific questionnaire: The "Minnesota Living With Heart Failure Questionnaire" (MLWHFQ). Blood analyses, ecocardiographic measurements and specific questionnaires, were also carried out in order to evaluate other parameters which could affect QL: Age, sex, comorbilidity, number of drugs taken, left ventricular ejection fraction, nutritional status, cognitive function, depression and functional capacity in daily life.

Results: $Q L$ measured by means of the $M L W H F Q$ was quite high (mean: 34.3). The other tests yielded values within normality or next to normality. Depression was the factor more clearly associated with patients' quality of life $(p=0.0001)$. We also found a statistically significant association with left ventricular ejection fraction $(p=0.03)$ age $(p=0.008)$ and albuminemia $(p=0.01)$.

Conclusions: In our group of patients, $Q L$ was conditioned by depression, left ventricular ejection fraction, age and albuminemia.

KEY WORDS: Heart failure. Quality of life. Minnesota.

Exteberría-Lekuona D, Sánchez-Álvarez J, Alonso Gutiérrez A, Acha Arrieta V, Campos Rivas R, Jarne Bertrán V. Estudio de la calidad de vida de pacientes con insuficiencia cardiaca en un servicio de Medicina Interna. An Med Interna (Madrid) 2007; $24:$ 57-60.

\section{INTRODUCCIÓN}

La insuficiencia cardiaca (IC) es una enfermedad crónica severa con gran prevalencia (1). Los pacientes con IC experimentan como consecuencia de su enfermedad limitaciones en la dieta, dificultades para realizar el trabajo habitual y para las relaciones sexuales, pérdida progresiva de la autonomía, ingresos hospitalarios frecuentes y efectos secundarios de los medicamentos. Todo ello produce un importante deterioro de su calidad de vida (CV) (2). Durante los últimos años, la CV de los pacientes con IC se ha convertido en un objetivo tan importante como la disminución de la mortalidad y el número de hospitalizaciones o la mejora de la función ventricular. Con frecuencia se han utilizado los cuestionarios de CV como medida del beneficio de una determinada intervención médica $(3,4)$. Además, la CV ha demostrado ser un predictor útil de mortalidad y de hospitalización $(5,6)$, y se ha convertido en un parámetro a tener en cuenta a la hora de tomar decisiones terapéuticas individualizadas.

Existen varios cuestionarios de CV específicos para pacientes con IC. Uno de los más utilizados es el Minnesota Living With Heart Failure Questionnaire (MLWHFQ) (7-8),

Trabajo aceptado: 6 de octubre de 2006 
que ha sido validado en varios países incluido EE.UU. En España, aunque no está validado en castellano, ya ha sido utilizado (8), constatándose una buena correlación entre la puntuación obtenida en el cuestionario y la clase funcional, el número de reingresos, el sexo, la edad y la presencia de ciertas enfermedades (diabetes, anemia, cardiopatía valvular).

Los objetivos del presente estudio fueron, evaluar, utilizando el $M L W H F Q$, la $\mathrm{CV}$ en un grupo de pacientes con IC ingresados en un servicio de Medicina Interna, y además, evaluar los factores asociados a dicha CV.

\section{MATERIAL Y MÉTODOS}

Durante seis meses, desde septiembre del 2004 hasta marzo del 2005, se registraron los pacientes con IC ingresados en el Servicio de Medicina Interna del Hospital Virgen del Camino, un hospital terciario situado en Pamplona. De cara a realizar el estudio sobre una muestra de población lo suficientemente homogénea, que al mismo tiempo fuera prevalente, se hizo una selección de los pacientes con los siguientes criterios de inclusión: a) criterios diagnósticos de IC del estudio Framingham (9); b) grado funcional III-IV de la NYHA (New York Heart Association); c) edad superior a 64 años; d) Puntuación en el Mini-Mental Status Examination (10) superior a 16 puntos; e) ausencia de síndrome coronario agudo asociado; f) expectativa de vida inicial mayor a 6 meses; y g) ausencia de enfermedad que pudiera confundirse con la IC o que pudiera condicionar "per se" de forma severa la calidad de vida: Ictus y déficit residual importante, enfermedad respiratoria severa, insuficiencia renal terminal, cirrosis, obesidad extrema, hemoglobina $<9 \mathrm{~g} / \mathrm{dl}$, arteriopatía periférica en estadio III-IV, enfermedad tiroidea o suprarrenal significativa, proceso neoplásico con supervivencia a los 2 años menor del $90 \%$.

Antes de iniciar el estudio se informó a cada paciente y éste firmó el consentimiento informado, validado, al igual que el diseño del estudio, por la Comisión de Ética del hospital. Con la ayuda de uno de los autores (DE), el paciente completó los siguientes cuestionarios: a) Mini-Mental Status Examination; 2) versión en castellano del $M L W H F Q$ proporcionada por el Mapi Research Institute; 3) escala de Lawton y Brody para las actividades instrumentales de la vida diaria (11); 4) indice de Katz para las actividades básicas de la vida diaria (12); y 5) escala de depresión geriátrica de Yesavage (13). Las diplomadas en Dietética del Servicio de Dietética y Nutrición supervisaron la realización del cuestionario Mini Nutritional Assessment-Test (14). Además de los cuestionarios se completaron las siguientes fichas: a) datos demográficos y de comorbilidad; b) características de la IC, con la función sistólica medida mediante ecocardiograma; y c) parámetros antropométricos y determinaciones de laboratorio.

La variable resultado principal en nuestro estudio era la puntuación en el cuestionario MLWHFQ. Cuanto mayor es la puntuación obtenida peor es la $\mathrm{CV}$ de los pacientes (rango de puntuación de 0 a 105 puntos). Se estudió la asociación existente entre dicha puntuación y diversas variables: Sexo, edad, hemoglobinemia, albuminemia, colesterolemia, valor de la fracción de eyección del ventrículo izquierdo (FEVI) medida mediante ecocardiograma, tipo de IC [Sistólica/Función Sistólica Preservada (FSP)], Grado Funcional de la NYHA (III/IV), comorbilidad expresada como número de enfermedades concomitantes, y número total de medicamentos que toma el paciente (en conjunto, y seleccionando sólo los que son específicos para el tratamiento de la IC y los factores de riesgo cardiovascular: AAS, hipolipemiantes, insulina, antidiabéticos orales, antiacoagulación, beta-bloqueantes, diuréticos, IECA, ARAII, calcio-antagonistas, alfa-bloqueantes, nitratos, vasodilatadores arteriales). También se estudio la asociación existente entre la puntuación obtenida en el $M L W H F Q$ y la obtenida en tests específicos para determinados parámetros condicionantes de la CV: Mini-Mental Status Examination, Escala de Lawton y Brody, Indice de Katz, Escala de depresión de Yesavage y Mini Nutritional Assessment. Para realizar estos estudios se utilizaron test no paramétricos. Si la variable independiente era dicotómica se empleó el test de la U de Mann-Whitney, y si la variable independiente era cuantitativa se utilizaron coeficientes de correlación de Spearman.

Por último, para estudiar de manera simultánea la asociación entre varias variables y la CV medida con el $M L W H F Q$, se empleó un modelo de regresión lineal múltiple, utilizando un algoritmo stepwise de selección automática de variables, con probabilidades de entrada y de salida de 0.15 . Se utilizó el programa estadístico SPSS v 10.0 (SPSS Inc, Chicago, IL, EE.UU.). Se consideraron valores significativos aquellos con un valor de $\mathrm{p}<0,05$.

\section{RESULTADOS}

En los 6 meses que duró la recogida de datos se produjeron 114 casos de IC, de los que 34 cumplieron los criterios de

\begin{tabular}{|c|c|}
\hline \multicolumn{2}{|c|}{ TABLA I } \\
\hline \multicolumn{2}{|c|}{ RESULTADOS DESCRIPTIVOS } \\
\hline Edad (años) & $79,7(6,1)$ \\
\hline \multicolumn{2}{|l|}{ Sexo $[n(\%)]$} \\
\hline Femenino & $18(52,9)$ \\
\hline Masculino & $16(47,1)$ \\
\hline Comorbilidad & $5,1(2,0)$ \\
\hline Medicación 1 & $5,9(2,6)$ \\
\hline Medicación 2 & $3,5(1,9)$ \\
\hline MMSE & $22,7(2,8)$ \\
\hline MLWHFQ & $34,3(23,1)$ \\
\hline Yesavage & $3,5(3,3)$ \\
\hline Lawton & $5,3(2,1)$ \\
\hline Katz & $0,45(1,03)$ \\
\hline FEVI & $59,4(16,4)$ \\
\hline MNA & $24,2(3,4)$ \\
\hline Hemoglobina & $12,8(2,1)$ \\
\hline Albúmina & $37,6(5,0)$ \\
\hline Colesterol & $166,3(43,9)$ \\
\hline \multicolumn{2}{|l|}{ Tipo de IC [n (\%)] } \\
\hline Sistólica & $5(14,7)$ \\
\hline FSP (FEVI > 40\%) & $29(85,3)$ \\
\hline \multicolumn{2}{|l|}{ GF NYHA [n (\%)] } \\
\hline III & $17(50,0)$ \\
\hline IV & $17(50,0)$ \\
\hline
\end{tabular}

En las variables cuantitativas los valores representan medias y desviaciones estándar. En las variables cualitativas se representan frecuencias [n (\%)] Medicación específica para la IC o los factores de riesgo cardiovascular. MMSE: mini-mental status examination; FEVI: fracción de eyección del ventrículo Izquierdo; MNA: mini nutritional assessment; FSP: función sistólica preservada; GF NYHA: grado funcional según la New York Heart Association. 
inclusión y aceptaron participar en el estudio. La tabla I muestra sus características. Se trataba de una muestra con una edad media alta, equilibrada en cuanto al sexo, en la que los pacientes tenían varias enfermedades concomitantes, estaban polimedicados, presentaban un deterioro cognitivo leve, la mayoría de ellos no estaban deprimidos, poseían una capacidad funcional bastante bien conservada y estaban bien nutridos. La fracción de eyección del ventrículo izquierdo estaba conservada en al mayoría de los casos $(85,3 \%)$. La puntuación obtenida en el MLWHFQ fue relativamente baja, con una media de 34,3 puntos (desviación estándar: 23,1), lo que implica una CV aceptable.

Se observó una asociación directa significativa entre la puntuación en el Yesavage y el $M L W H F Q$ (coeficiente de

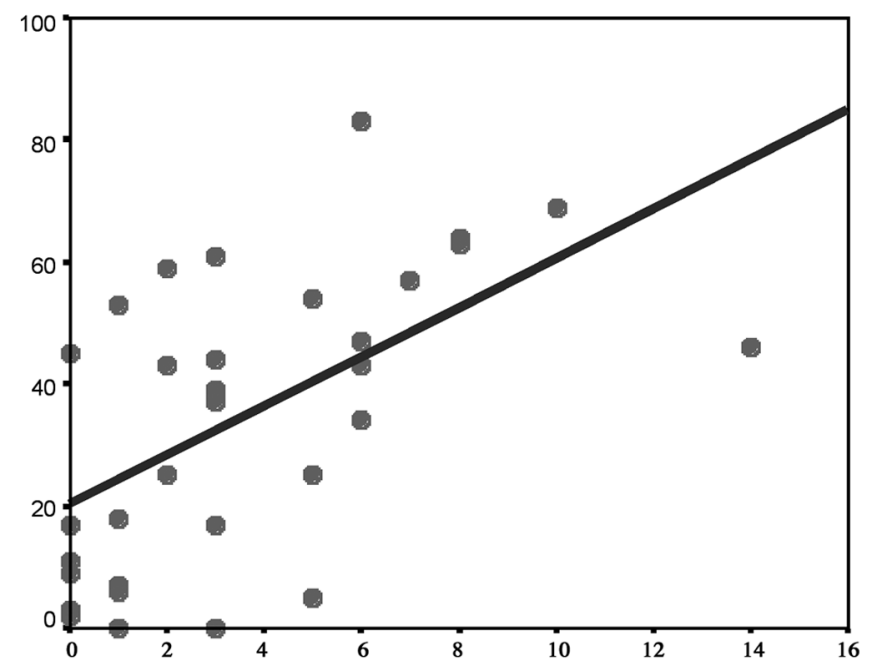

Fig. 1. Asociación entre la escala de Yesavage y la puntuación en el MLWHFQ ("Coeficiente de correlación de Spearman") rho =0,61, $p=0,0001$ ).

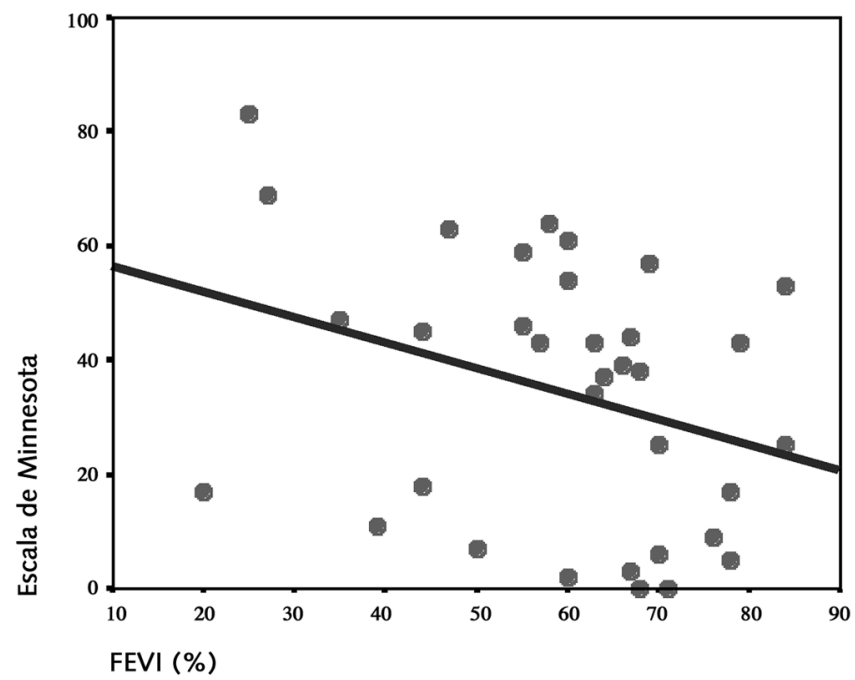

Fig. 2. Asociación entre la FEVI y puntuacion en el MLWHFQ. correlación rho de Spearman $=0,61, p=0,0001)($ Fig. 1). Este resultado suponía que cuanto más deprimido estaba el paciente (mayor puntuación en la Escala de Yesavage), menor era su CV (mayor puntuación en el MLWHFQ). Se observó también una asociación inversa significativa entre el valor de la FEVI y el MLWHFQ (coeficiente de correlación rho de Spearman = $-0,38, p=0,03$ ) (Fig. 2). En el análisis multivariante se objetivó una asociación estadísticamente significativa de la Escala de Yesavage, la edad y los niveles de albúmina con la puntuación obtenida en el MLWHFQ (Tabla II).

\begin{tabular}{lccc}
\multicolumn{4}{c}{ TABLA II } \\
\hline Variable & Coeficiente & \multicolumn{1}{c}{ ANÁLISIS MULTIVARIANTE } \\
\hline Yesavage & 5.76 & $3,65,7,88$ & $P$ \\
Edad & 1,33 & $0,43,2,23$ & 0,0001 \\
Albuminemia & $-1,92$ & $-3,28,-0,57$ & 0,01 \\
Comorbilidad & $-2,40$ & $-5,18,0,38$ & 0,10 \\
FEVI & $-0,29$ & $-0,66,0,09$ & 0,15
\end{tabular}

Asociación entre diversas variables y el nivel de calidad de vida valorado mediante el MLWHFQ. Resultados de un modelo de regresión multivariable con el valor de MLWHFQ como variable dependiente, y las variables representadas en la tabla I como variables predictoras.

\section{DISCUSIÓN}

La CV media cuantificada mediante el MLWHFQ en la población estudiada era buena (34,3 puntos), y parecida a la obtenida por la serie de Parajón y cols. (8). Esta circunstancia se puede justificar porque el resto de parámetros que podían haber influido negativamente (Mini-Mental Status Examination, Yesavage, Lawton, Katz, MNA, hemoglobinemia, albúminemia, colesterolemia) presentaban valores dentro de la normalidad o próximos a ella (Tabla I).

La depresión era el factor que se relacionaba con mayor intensidad con la CV de los pacientes (Fig. 2 y Tabla II). Éste es un factor que ya había demostrado tener una clara influencia en la CV de este tipo de pacientes en estudios previos (15). La segunda variable que de forma aislada demostró una relación estadísticamente significativa con la CV medida mediante el $M L W H F Q$ fue la FEVI (Fig. 2). La correlación era inversa y de intensidad moderada. Ello suponía que cuanto mayor era la FEVI menor era la puntuación en el $M L W H F Q$ (mejor CV). La edad presentaba una asociación directa con la CV medida mediante el MLWHFQ que era estadísticamente significativa en el análisis multivariante (Tabla II). También el nivel de albúmina en sangre presentaba una asociación estadísticamente significativa con la puntuación en el MLWHFQ, pero la correlación era inversa (Tabla II).

A la hora de evaluar los resultados se objetivaron ciertas limitaciones relacionadas con la comorbilidad de los pacientes y el tamaño de la muestra. Al diseñar el estudio se tuvieron en cuenta las enfermedades concomitantes que podían actuar como factor de confusión, lo que motivó que un importante número de pacientes no fuera incluido en el estudio. Debido a ello la muestra estudiada en este trabajo es pequeña $(n=34)$, lo que limita la potencia del mismo. Para solventar estos pro- 
blemas y obtener resultados más concluyentes, sería aconsejable una muestra mayor de pacientes.

En conclusión, en nuestro estudio la depresión fue el factor que con más claridad se asoció con la $\mathrm{CV}$ de los pacientes con
IC. También demostraron una asociación estadísticamente significativa entre la FEVI, la edad y la albuminemia. En el futuro, para valorar la CV de los pacientes con IC, sería interesante diseñar estudios como el presente pero con carácter multicéntrico.

\section{Bibliografía}

1. Rodriguez-Artalejo F, Banegas JR, Guallar-Castillón P. Epidemiología de la insuficiencia cardiaca. Rev Esp Cardiol 2004; 57: 163-170.

2. Mc Murray JJ, Petrie MC, Murdoch DR, Davie AP. Clinical epidemiology of heart failure: public and private health burden. Eur Heart J 1998; 19 (Supl. P): 9-12.

3. Majani G, Giardini A, Opasich C, Glazer R, Hester A, Tognoni G, et al. Effect of valsartan on quality of life when added to usual therapy for heart failure: Results from the Valsartan Heart Failure Trial. J Card Fail 2005 Jun; 11 (5): 404.

4. Rector TS, Kubo SH, Cohn JN.. Validity of the Minnesota living with heart failure questionnaire as a measure of therapeutic response to enalapril or placebo. Am J Cardiol 1993; 71: 1106-7.

5. Rodríguez-Artalejo F, Guallar-Castillon P, Pascual CR, Otero CM, Montes AO, García AN, et al. Health-related quality of life as a predictor of hospital readmission and death among patients with heart failure. Arch Intern Med 2005 Jun 13; 165: 1274-9.

6. Alla F, Briançon S, Guillemin F, Jullière Y, Mertès PM, Villemot JP, et al, for the EPICAL investigators. Self-rating of quality of live provides additional prognostic information in heart failure. Insights into the EPICAL study. Eur J Heart Failure 2002; 4: 337-43.

7. Rector TS, Kubo SH, Cohn JN. Patients self assessment of their congestive heart failure: II. Content, reliability and validity of a new measurethe Minnesota Living with Heart Failure questionnaire. Heart Failure
1987; 3: 198-209.

8. Parajón T, Lupón J, González B, et al. Aplicación en España del cuestionario sobre calidad de vida "Minnesota Living With Heart Failure" para la insuficiencia cardiaca. Rev Esp Cardiol 2004; 57: 155-60.

9. Ho KK, Anderson KM, Kannel WB. Survival after the onset of congestive heart failure in Framingham Heart Study subjets. Circulation 1993; 88: $107-15$

10. Cockrell JR and Folstein MF. Mini Mental State Examination (MMSE), Psychopharmacology 1988; 24: 689-92.

11. Lawton MP, Brody EM. Assessment of older people: self-maintaining and instrumental activities of daily living. Gerontologist, 1969; 9: 179-186.

12. Katz, S, Ford AB, Moscowitz RW, Jackson BA, Jaffe MW. Studies of illness in the aged. The index of ADL: a standarized measure of biological and psychosocial function. Jama 1963; 185: 914-9.

13. Yesavage JA, Brink TL, Rose TL, Lum O, Huang V, Adey M, et al. Development and validation of a geriatric depression screening scale: a preliminary report. J Psychiatr Res 1983; 17: 37-49.

14. Guigoz Y, Vellas B, Garry PJ. Mini Nutritional Assessment: A practical assessment tool for grading the nutritional state of elderly patients. Facts Res Gerontol 1994; (Supl. 2): 15-59.

15. Carels RA. The association between disease severity, functional status, depression and daily quality of life in congestive heart failure patients. Qual Life Res 2004; 13: 63-72. 